\title{
Adaptive Measurement Matrix Design for Compressed DoA Estimation with Sensor Arrays
}

\author{
Berk Ozer $^{\ddagger}$, Anastasia Lavrenko ${ }^{\dagger}$, Sinan Gezici ${ }^{\ddagger}$, Florian Römer ${ }^{\dagger}$, Giovanni Del Galdo ${ }^{\dagger}$ and Orhan Arikan ${ }^{\ddagger}$ \\ ${ }^{\dagger}$ Institute for Information Technology, Technische Universität Ilmenau \\ Helmholzplatz 2, 98693, Ilmenau, Germany \\ ${ }_{\ddagger}^{\ddagger}$ Electrical and Electronics Engineering Department, Bilkent University \\ Bilkent, TR-06800, Ankara, Turkey \\ Contact email: ozer@ee.bilkent.edu.tr,+90 5336839342
}

\begin{abstract}
In this work we consider the problem of measurement matrix design for compressed 3-D Direction of Arrival (DoA) estimation using a sensor array with analog combiner. Since generic measurement matrix designs often do not yield optimal estimation performance, we propose a novel design technique based on the minimization of the Crameŕ-Rao Lower Bound (CRLB). We develop specific approaches for adaptive measurement design for two applications: detection of the newly appearing targets and tracking of the previously detected targets. Numerical results suggest that the developed designs allow to provide the near optimal performance in terms of the CRLB.
\end{abstract}

\section{INTRODUCTION}

In recent years, the newly emerged measurement paradigm of Compressive sensing (CS) has been successfully applied to sensor array processing problems [1-3]. The application of CS allows to provide estimation performance comparable to the classical techniques by using fewer number of sensors that is traditionally required. A variety of array configurations practically implementing such spatial compression has been proposed in the literature. One particular approach is to combine the $N$ array outputs into only $M<N$ receiver channels [4]. Obviously, the choice of the combining weights, i.e., the so-called measurement matrix, plays a crucial role in resulting DoA estimation performance as well as in system design. It has been recently demonstrated that typically considered in the CS related works random measurement matrix designs are not necessarily optimal for particular signal processing tasks [5], [6]. Therefore different approaches for measurement matrix optimization have been proposed in the literature.

One of the common ways to design the measurement matrix is by minimizing various forms of mutual coherence between its columns [7], [8]. However, since the mutual coherence accounts for the worst-case performance only, it does not characterize overall Dorection of Arrival (DoA) estimation accuracy. Another approach is to design the measurement matrix for specific task-driven purposes, e.g., to improve classification or estimation performance within a compressive framework as in [5], [9]. Thus, in [6] a design approach based on the matrix optimization with regard to the spatial correlation function of the resulting effective array manifold is proposed, whereas a design minimizing the Cramer-Rao Lower Bound (CRLB) is discussed in [10], [11]. One of the advantages of the CRLB minimization based design is that it provides an optimization framework which is independent of the estimator.

Motivated by the latter, in this work we propose a novel measurement matrix design technique based on the minimization of the CRLB. Our method generalizes the single parameter case from [10] to the multi-parameter case within a more comprehensive framework. We analytically show that the proposed measurement matrix allows to achieve the lowest CRLB possible. Using the proposed technique, we develop concrete algorithms for adaptive measurement design for two distinct DoA estimation modes: detection of the newly appearing targets (surveillance) and tracking of the previously detected targets (target tracking). In the surveillance mode, we first split the search space into several sectors and then iteratively update the measurement matrix within each of them. In the target tracking mode, we use the predicted position of a target obtained from the past estimates to adaptively design measurement matrix for the next estimation step. The results of the numerical simulations demonstrate that the proposed algorithms yield near-optimum results compared to the derived lower bounds and provide a significant improvement over the random measurement matrix designs.

The remainder of the paper is organized as follows. In Section II, we introduce the DoA estimation signal model and explain the compressive sensor array with analog combiner. The proposed measurement matrix optimization approach is presented in Section III. In Section IV, we discuss the adaptive measurement design for signal surveillance and target tracking. Finally, in Sections V and VI we present some numerical results and conclude the paper, respectively.

\section{SignAl MODEL}

Suppose that $\chi_{k}=\left[\begin{array}{ll}\theta_{k} & \phi_{k}\end{array}\right]$ denotes the angular orientation of a plane wave impinging from the $k^{\text {th }}$ target on some $N$ element array, where $\theta_{k} \in\left[0, \frac{\pi}{2}\right]$ and $\phi_{k} \in[0, \pi]$ represent the elevation and azimuth angles, respectively. In the presence of $K$ targets in the far field of the array, the signal (baseband) received at the $n^{\text {th }}$ sensor is given by

$$
x_{n}(t)=\sum_{k=1}^{K} s_{k} \cdot e^{j \omega\left(t-\tau_{n}\left(\chi_{k}\right)\right)},
$$


where $\omega$ is the carrier frequency, $s_{k}$ is the complex amplitude and $\tau_{n}\left(\chi_{k}\right)$ is relative time delay of the signal impinging from direction $\chi_{k}$. The value of $\tau_{n}\left(\chi_{k}\right)$ can be calculated as

$$
\tau_{n}\left(\boldsymbol{\chi}_{k}\right)=\frac{\boldsymbol{P}_{n}^{T}}{c}\left[\begin{array}{c}
-\sin \left(\theta_{k}\right) \cos \left(\phi_{k}\right) \\
-\sin \left(\theta_{k}\right) \sin \left(\phi_{k}\right) \\
-\cos \left(\theta_{k}\right)
\end{array}\right],
$$

where $\boldsymbol{P}_{n}^{\mathrm{T}}$ is the vector of relative positions of $n^{\text {th }}$ sensor with respect to the reference point and $c$ denotes the speed of light.

To reduces the number of channels to be sampled from $N$ to $M(M<N)$ we employ an analog pre-combiner at the sensor outputs as depicted in Figure 1. This allows us to decrease the number of ADCs and the amount of data to be processed while preserving a larger aperture. Denoting $y_{m}(t)$ the signal at the $m^{\text {th }}$ output of such a combiner, we have that

$$
y_{m}(t)=\sum_{n=1}^{N} w_{m n} \cdot\left(x_{n}(t)+n(t)\right),
$$

where $w_{m n}$ is the weight of the $n^{\text {th }}$ sensor in the $m^{\text {th }}$ combiner channel and $n(t)$ is the circularly symmetric Gaussian noise with variance $\sigma_{n}^{2}$.

Stacking the channel weights $w_{m n}$ into an $M \times N$ matrix $\boldsymbol{W}$, we obtain the following expression for the array output after sampling

$$
\boldsymbol{y}\left[t_{i}\right]=\boldsymbol{W} \tilde{\boldsymbol{x}}\left[t_{i}\right]=\boldsymbol{W}\left(\boldsymbol{x}\left[t_{i}\right]+\boldsymbol{n}\left[t_{i}\right]\right),
$$

where $\boldsymbol{y}\left[t_{i}\right]$ is an $M \times 1$ vector of measurements, $t_{i}$ is some sampling time and $\tilde{\boldsymbol{x}}\left[t_{i}\right]$ is an $N \times 1$ vector containing sampled equivalents of $N$ sensor outputs.

Collecting $L$ consequent measurements $\boldsymbol{y}\left[t_{i}\right], i=$ $1,2, \cdots, L$, into an $M \times L$ matrix $\boldsymbol{Y}$, we have that

$$
\boldsymbol{Y}=\boldsymbol{W} \cdot \tilde{\boldsymbol{X}}=\boldsymbol{W} \cdot(\boldsymbol{X}+\boldsymbol{N})
$$

where $\boldsymbol{X}$ and $\boldsymbol{N}$ are $N \times L$ matrices containing sampled equivalents of the input signal and the noise at the $N$ array outputs, respectively.

In this paper we aim at designing the measurement matrix $\boldsymbol{W}$ that minimizes the CRLB for estimating the DOA of a single signal $(K=1)$ of known amplitude impinging from $\chi$ direction on the sensor array from Fig. 1.

\section{Measurement Matrix Design Based on CRLB MINIMIZATION}

\section{A. Objective Function}

We begin by defining our objective function as

$$
\begin{gathered}
\boldsymbol{W}^{*}(\boldsymbol{\chi})=\underset{\boldsymbol{W}}{\arg \min } \operatorname{Tr}\left\{\boldsymbol{J}^{-1}(\boldsymbol{W}, \boldsymbol{\chi})\right\} \\
\text { s.t. } \boldsymbol{W} \boldsymbol{W}^{\mathrm{H}}=\mathbf{I}_{M},
\end{gathered}
$$

where $\mathbf{I}_{M}$ denotes an $M \times M$ identity matrix and $\operatorname{Tr}\left\{\boldsymbol{J}^{-1}(\boldsymbol{W}, \boldsymbol{\chi})\right\}$ refers to the trace of the inverse Fisher Information matrix evaluated at $\chi$. The constraint $\boldsymbol{W} \boldsymbol{W}^{\mathrm{H}}=$

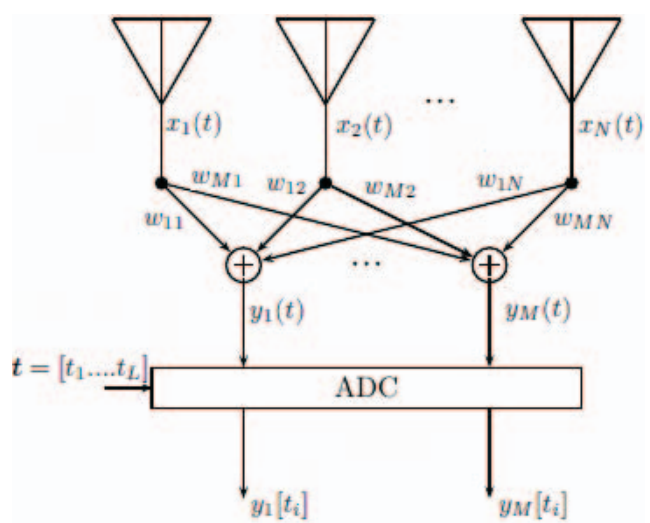

Fig. 1. Compressive sensor array with analog pre-combiner.

$\mathbf{I}_{M}$ in (6) ensures that $\boldsymbol{W}$ is full rank, i.e., it provides nonredundant set of measurements, and avoids coloring the noise ${ }^{1}$. For the $\operatorname{Tr}\left\{\boldsymbol{J}^{-1}(\boldsymbol{W}, \boldsymbol{\chi})\right\}$ we can write

$$
\operatorname{Tr}\left\{\boldsymbol{J}^{-1}(\boldsymbol{W}, \boldsymbol{\chi})\right\}=c_{0} \frac{e_{\theta}(\boldsymbol{W}, \boldsymbol{\chi})+e_{\phi}(\boldsymbol{W}, \boldsymbol{\chi})}{e_{\theta}(\boldsymbol{W}, \boldsymbol{\chi}) e_{\phi}(\boldsymbol{W}, \boldsymbol{\chi})-\left(e_{\theta \phi}(\boldsymbol{W}, \boldsymbol{\chi})\right)^{2}},
$$

where $c_{0}$ is a constant associated with the signal-to-noise ratio (SNR) and $e_{\theta}(\boldsymbol{W}, \boldsymbol{\chi}), e_{\phi}(\boldsymbol{W}, \boldsymbol{\chi})$ are the terms associated with the mean square errors for $\theta$ and $\phi$, respectively. It is shown in the Appendix, that

$$
\begin{aligned}
e_{\theta}(\boldsymbol{W}, \boldsymbol{\chi}) & =\boldsymbol{a}(\boldsymbol{\chi})^{\mathrm{H}} \boldsymbol{W}^{\mathrm{H}} \boldsymbol{W} \boldsymbol{a}(\boldsymbol{\chi}), \\
e_{\phi}(\boldsymbol{W}, \boldsymbol{\chi}) & =\boldsymbol{b}(\chi)^{\mathrm{H}} \boldsymbol{W}^{\mathrm{H}} \boldsymbol{W} \boldsymbol{b}(\chi), \\
e_{\theta \phi}(\boldsymbol{W}, \boldsymbol{\chi}) & =\boldsymbol{a}(\chi)^{\mathrm{H}} \boldsymbol{W}^{\mathrm{H}} \boldsymbol{W} \boldsymbol{b}(\boldsymbol{\chi}),
\end{aligned}
$$

where $\boldsymbol{a}(\boldsymbol{\chi})=\frac{\partial e^{j \omega \boldsymbol{\tau}(\boldsymbol{\chi})}}{\partial \theta}$ and $\boldsymbol{b}(\boldsymbol{\chi})=\frac{\partial e^{j \omega \boldsymbol{\tau}(\boldsymbol{\chi})}}{\partial \phi}$. Note that it can be shown that for circular arrays $\boldsymbol{a}(\chi) \perp \boldsymbol{b}(\chi)$ (see Appendix).

\section{B. Optimal Measurement Design}

Proposition 1. For a measurement matrix $\boldsymbol{W}$ that satisfies $\boldsymbol{W} \boldsymbol{W}^{\mathrm{H}}=\mathbf{I}_{M}, L(\boldsymbol{W}, \boldsymbol{\chi})=\frac{1}{\|\boldsymbol{a}(\boldsymbol{\chi})\|^{2}}+\frac{1}{\|\boldsymbol{b}(\boldsymbol{\chi})\|^{2}}$ provides $a$ lower bound for the CRLB:

$$
\frac{\operatorname{Tr}\left\{\boldsymbol{J}^{-1}\left(\boldsymbol{W}^{*}(\boldsymbol{\chi}), \boldsymbol{\chi}\right)\right\}}{c_{0}} \geq L(\boldsymbol{W}, \boldsymbol{\chi}) .
$$

Proof. Suppose $\boldsymbol{W}^{\mathrm{H}}=\left[\widehat{\boldsymbol{w}}_{1}^{*}, \widehat{\boldsymbol{w}}_{2}^{*}, \cdots, \widehat{\boldsymbol{w}}_{M}^{*}\right]$, where $\left\{\widehat{\boldsymbol{w}}_{1}, \cdots, \widehat{\boldsymbol{w}}_{M}\right\}$ is an orthonormal set, i.e. $\left\|\widehat{\boldsymbol{w}}_{m}\right\|^{2}=1$ and $\widehat{\boldsymbol{w}}_{i}^{\mathrm{H}} \widehat{\boldsymbol{w}}_{j}=0 \forall i \neq j$. Then, for the left part of (11) we can write

\footnotetext{
${ }^{1}$ Note that this constraint can be imposed on $\boldsymbol{W}$ without loss of generality since it can be shown that for every non-orthogonal $\boldsymbol{W}$ we can find a corresponding orthogonal one that achieves the same CRLB.
} 


$$
\begin{aligned}
\frac{\operatorname{Tr}\left\{\boldsymbol{J}^{-1}(\boldsymbol{W}(\boldsymbol{\chi}), \boldsymbol{\chi})\right\}}{c_{0}} & \geq \frac{e_{\theta}(\boldsymbol{W}, \boldsymbol{\chi})+e_{\phi}(\boldsymbol{W}, \boldsymbol{\chi})}{e_{\theta}(\boldsymbol{W}, \boldsymbol{\chi}) e_{\phi}(\boldsymbol{W}, \boldsymbol{\chi})} \\
& =\frac{1}{e_{\theta}(\boldsymbol{W}, \boldsymbol{\chi})}+\frac{1}{e_{\phi}(\boldsymbol{W}, \boldsymbol{\chi})} \\
& =\frac{1}{\sum_{m=1}^{M}\left|\boldsymbol{a}(\boldsymbol{\chi})^{\mathrm{H}} \widehat{\boldsymbol{w}}_{m}\right|^{2}}+\frac{1}{\sum_{m=1}^{M}\left|\boldsymbol{b}(\boldsymbol{\chi})^{\mathrm{H}} \widehat{\boldsymbol{w}}_{m}\right|^{2}} \\
& \geq \frac{1}{\|\boldsymbol{a}(\boldsymbol{\chi})\|^{2}}+\frac{1}{\|\boldsymbol{b}(\boldsymbol{\chi})\|^{2}} .
\end{aligned}
$$

The following theorem provides the solution to (6).

Theorem 1. Let $\widehat{a}(\chi)=\frac{a(\chi)}{\|\boldsymbol{a}(\chi)\|}$ and $\widehat{b}(\chi)=\frac{\boldsymbol{b}(\chi)}{\|\boldsymbol{b}(\chi)\|}$. Then, $\boldsymbol{W}^{*}(\chi)=\left[\begin{array}{l}\widehat{\boldsymbol{a}}(\chi)^{\mathrm{H}} \\ \widehat{\boldsymbol{b}}(\chi)^{\mathrm{H}}\end{array}\right]$ is the optimal measurement matrix that minimizes CRLB.

Proof. To prove Theorem 1, we will show that the measurement matrix $\boldsymbol{W}^{*}(\chi)=\left[\begin{array}{l}\widehat{\boldsymbol{a}}(\chi)^{\mathrm{H}} \\ \widehat{b}(\chi)^{\mathrm{H}}\end{array}\right]$ allows to achieve $L(\boldsymbol{W}, \boldsymbol{\chi})$, which, according to the Proposition 1 , is the lower bound for the estimation error. To do so we write (8) as

$$
\begin{aligned}
& e_{\theta}\left(\boldsymbol{W}^{*}(\chi), \chi\right)=\boldsymbol{a}(\chi)^{\mathrm{H}}\left[\begin{array}{ll}
\widehat{\boldsymbol{a}}(\chi) & \widehat{\boldsymbol{b}}(\chi)^{\mathrm{H}}
\end{array}\right]\left[\begin{array}{l}
\widehat{a}(\chi)^{\mathrm{H}} \\
\widehat{b}(\chi)^{\mathrm{H}}
\end{array}\right] \boldsymbol{a}(\chi) \\
& =\boldsymbol{a}(\chi)^{\mathrm{H}}\left(\widehat{\boldsymbol{a}}(\chi) \widehat{\boldsymbol{a}}(\chi)^{\mathrm{H}}+\widehat{\boldsymbol{b}}(\chi) \widehat{\boldsymbol{b}}(\chi)^{\mathrm{H}}\right) \boldsymbol{a}(\chi) \\
& =\frac{\left(\boldsymbol{a}(\chi)^{\mathrm{H}} \boldsymbol{a}(\chi)^{\mathrm{H}}\right)^{2}}{\boldsymbol{a}(\chi)^{\mathrm{H}} \boldsymbol{a}(\chi)^{\mathrm{H}}}=\|\boldsymbol{a}(\chi)\|^{2},
\end{aligned}
$$

since $\boldsymbol{b}(\chi)^{\mathrm{H}} \boldsymbol{a}(\chi)=0$ (see App.). Following the same procedure, it is easy to show that

$$
\begin{aligned}
e_{\phi}\left(\boldsymbol{W}^{*}(\chi), \chi\right) & =\|\boldsymbol{b}(\chi)\|^{2}, \\
e_{\theta \phi}\left(\boldsymbol{W}^{*}(\chi), \chi\right) & =0 .
\end{aligned}
$$

Substituting (12), (13) and (14) into (7) we have that

$$
\begin{aligned}
\operatorname{Tr}\left\{\boldsymbol{J}^{-1}\left(\boldsymbol{W}^{*}(\chi), \chi\right)\right\} & =c_{0} \frac{\|\boldsymbol{a}(\boldsymbol{\chi})\|^{2}+\|\boldsymbol{b}(\boldsymbol{\chi})\|^{2}}{\|\boldsymbol{a}(\boldsymbol{\chi})\|^{2}\|\boldsymbol{b}(\boldsymbol{\chi})\|^{2}} \\
& =c_{0}\left(\frac{1}{\|\boldsymbol{a}(\boldsymbol{\chi})\|^{2}}+\frac{1}{\|\boldsymbol{b}(\boldsymbol{\chi})\|^{2}}\right) .
\end{aligned}
$$

Theorem 1 also illustrates that it is sufficient to use only the measurement matrix with only 2 rows, i.e., $M=2$, to reach the optimal performance in terms of the CRLB.

\section{Sensor Array Processing Applications}

\section{A. Surveillance}

In the surveillance mode we want to detect new targets possibly emerging into the scene. In order to survey the entire search region using the proposed design, we partition the surveillance region, i.e., $\mathcal{S}=\left[0, \frac{\pi}{2}\right] \times[0, \pi]$, into a

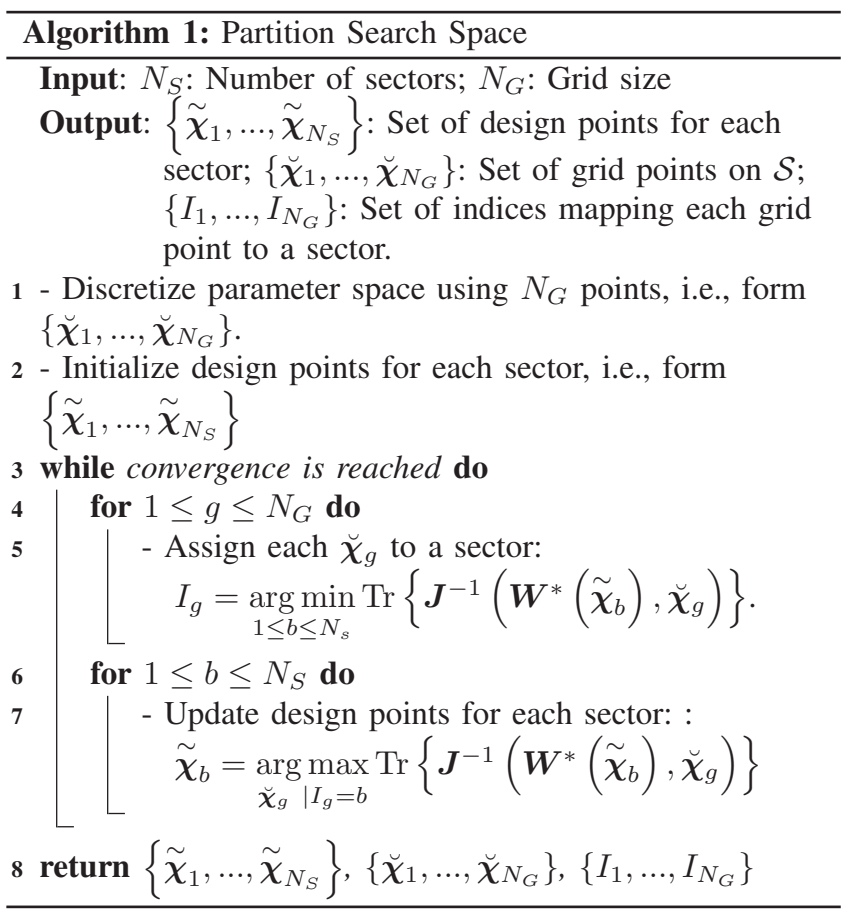

set of $N_{S}$ sectors $S_{b}$, where $1 \leq b \leq N_{S}$. Surveillance in each sector is conducted by a measurement matrix $W^{*}\left(\tilde{\chi}_{b}\right)$, where $\tilde{\chi}_{b} \in S_{b}$.

A straightforward way of choosing $\tilde{\chi}_{b}$ for $b^{\text {th }}$ sector is to minimize the following worst case performance within $S_{b}$

$$
\tilde{\boldsymbol{\chi}}_{b}=\underset{\boldsymbol{\chi} \in S_{b}}{\arg \max } \operatorname{Tr}\left\{\boldsymbol{J}^{-1}\left(\boldsymbol{W}^{*}(\boldsymbol{\chi}), \boldsymbol{\chi}\right)\right\} .
$$

However, (16) requires that all $S_{b}, 1 \leq b \leq N_{S}$ are perfectly known. Therefore, we need to consider a following optimization problem instead

$$
\begin{aligned}
& \min _{S_{b}} \max _{\boldsymbol{\chi} \in S_{b}} \operatorname{Tr}\left\{\boldsymbol{J}^{-1}\left(\boldsymbol{W}^{*}(\boldsymbol{\chi}), \boldsymbol{\chi}\right)\right\} \\
& \text { s.t. } \mathcal{S}=S_{1} \cup S_{2} \cup \ldots \cup S_{N_{S}}, \\
& S_{b} \cap S_{j}=\emptyset \forall b \neq j .
\end{aligned}
$$

To find a solution to (17) providing both the set of sectors $\left(\left\{S_{1}, \ldots, S_{N_{S}}\right\}\right)$ and corresponding design points $\left(\left\{\tilde{\chi}_{1}, \ldots, \tilde{\chi}_{N_{S}}\right\}\right)$, we develop an iterative algorithm inspired by the vector quantization approaches such as K-Means [12]. As summarized in Algorithm 1, we first discretize the search space by a set of $N_{G}$ grid points, $\left\{\breve{\chi}_{1}, \ldots, \breve{\chi}_{N_{G}}\right\}$, and initial design points, $\left\{\tilde{\chi}_{1}, \ldots, \tilde{\chi}_{N_{S}}\right\}$. After initialization, we form sectors by assigning each grid point to the design point associated with a sector that yields the best performance. Then, we update design points of the resulting sectors so that we improve the worst case performance of each sector. We iteratively perform these 2 steps until we reach the convergence.

\section{B. Tracking}

Once a newly emerged target is detected in the surveillance mode, the corresponding parameters of the target are passed 


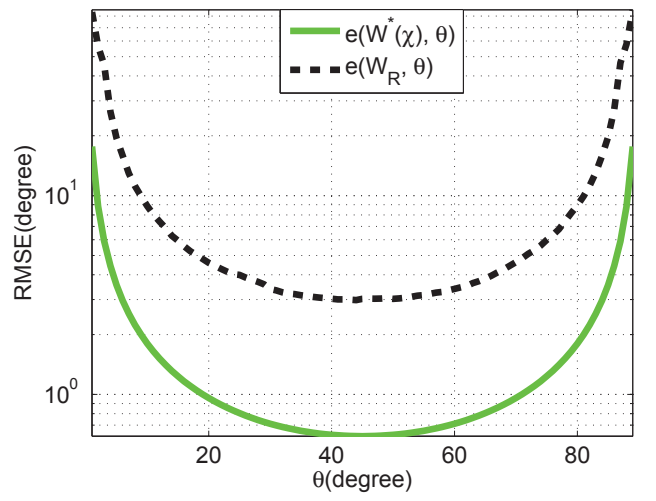

Fig. 2. Average RMSE-CRLB as a function of $\theta$ for the proposed measurement matrix design (green) and random Gaussian matrix (black).

to the tracker. In the tracking mode the predicted position $\widehat{\chi}^{i \mid i-1}$ of a tracked target can be obtained by using a proper target model and past estimates. We adaptively design the measurement matrix $\boldsymbol{W}^{*}\left(\widehat{\chi}^{i \mid i-1}\right)$, e.g., using the approach from Section III-B, based on the previous estimate of the target position.

\section{Simulation Results}

\section{A. Performance Analysis}

To evaluate the performance of the proposed measurement matrix design technique, we use uniform circular array (UCA) with $N=24$ sensors which we compress to $M=2$ outputs. The signal-to-noise ratio (SNR), i.e., $|s|^{2} / \sigma_{0}^{2}$, is set to $3 \mathrm{~dB}$. Since the UCA is known to be equally sensitive to all azimuth angles $\phi$, we can represent $\operatorname{Tr}\left\{\boldsymbol{J}^{-1}(\boldsymbol{W}, \boldsymbol{\chi})\right\}$ as a function of $\theta$ and $\boldsymbol{W}$ only, i.e., $\operatorname{Tr}\left\{\boldsymbol{J}^{-1}(\boldsymbol{W}, \boldsymbol{\chi})\right\} \equiv \operatorname{Tr}\left\{\boldsymbol{J}^{-1}(\boldsymbol{W}, \theta)\right\}$.

Denote $E(\boldsymbol{W}, \theta)$ the root-mean-square (RMSE)-CRLB measured in degrees such that

$$
E(\boldsymbol{W}, \theta)=\frac{180}{\pi} \sqrt{\operatorname{Tr}\left\{\boldsymbol{J}^{-1}(\boldsymbol{W}, \theta)\right\}}
$$

For various values of $\theta \in[0, \pi / 2]$, we calculate $E\left(\boldsymbol{W}^{*}(\chi), \theta\right)$ and $E\left(\boldsymbol{W}_{R}, \theta\right)$, where $\boldsymbol{W}_{R}$ denotes random matrices with entries drawn independently from normal Guassian distribution. In Figure 2, the resulting average values of $E\left(\boldsymbol{W}^{*}(\chi), \theta\right)$ and $E\left(\boldsymbol{W}_{R}, \theta\right)$ are shown. We can observe that the proposed measurement design allows to improve the DoA estimation accuracy compared to the common (random) Gaussian measurement matrix.

In order to investigate sensitivity of the proposed design to the choice of the design point, we introduce a following model

$$
\chi=\chi_{D}+\delta \chi
$$

where $\chi$ is an unknown true DoA, $\chi_{D}$ is a known design point and $\delta \chi \sim \mathcal{N}\left(\mathbf{0}, \sigma_{m}^{2}\right)$. We define the RMSE-CRLB for this model mismatch as

$$
E_{m}\left(\boldsymbol{W}, \theta, \sigma_{m}\right)=\mathbb{E}[E(\boldsymbol{W}, \theta)]
$$

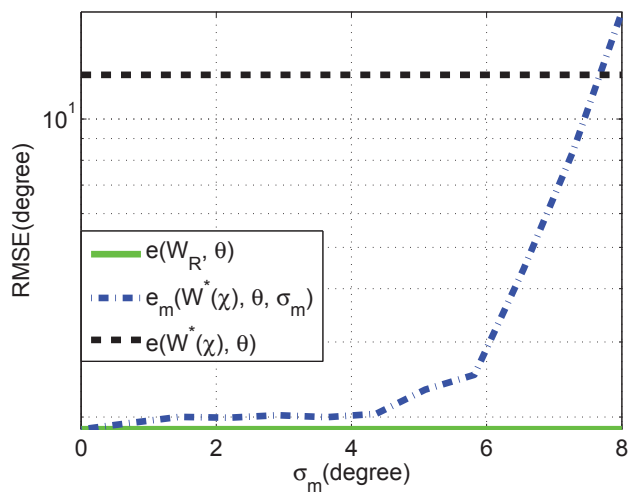

Fig. 3. Average RMSE-CRLB as a function of standard deviation of model mismatch.

where the expectation is computed over the parameter $\delta \chi$. Note that $E_{m}\left(\boldsymbol{W}_{R}, \theta, \sigma_{m}\right)=E\left(\boldsymbol{W}_{R}, \theta\right)$ because it is independent of $\delta \boldsymbol{\chi}$. In Fig. 3, we demonstrate $E\left(\boldsymbol{W}^{*}(\boldsymbol{\chi}), \theta\right)$, $E\left(\boldsymbol{W}_{R}, \theta\right)$ and $E_{m}\left(\boldsymbol{W}^{*}(\boldsymbol{\chi}), \theta, \sigma_{m}\right)$ as functions of $\sigma_{m}$. We observe that the proposed technique yields near-optimum results for smaller values of $\sigma_{m}$; as $\sigma_{m}$ increases, the performance starts to degrade. However, the random Gaussian matrix starts to outperform the proposed measurement matrix only after a relatively high value of $\sigma_{m}$, e.g., $\sigma_{m} \approx 7.5^{\circ}$.

\section{B. Surveillance}

Suppose that $\breve{\chi}_{g}$ is a point that belongs to the $b^{\text {th }}$ surveillance sector defined in Section IV-A. However, when operating in the surveillance mode we design the measurement matrix according to some sector design point $\tilde{\chi}_{b}$. Since the true DoA is not known in advance but has to be estimated, $\breve{\chi}_{g}$ might differ from $\tilde{\chi}_{b}$ significantly. Thus, we introduce the following metric

$$
0 \leq \mathrm{O}\left\{\breve{\boldsymbol{\chi}}_{g}\right\}=\frac{\operatorname{Tr}\left\{\boldsymbol{J}^{-1}\left(\boldsymbol{W}^{*}\left(\breve{\boldsymbol{\chi}}_{g}\right), \breve{\boldsymbol{\chi}}_{g}\right)\right\}}{\operatorname{Tr}\left\{\boldsymbol{J}^{-1}\left(\boldsymbol{W}^{*}\left(\tilde{\boldsymbol{\chi}}_{b} \mid I_{g}=b\right), \breve{\boldsymbol{\chi}}_{g}\right)\right\}} \leq 1
$$

where the enumerator provides the optimum performance, while the denominator accounts for the deviation between the sector design point and the true DoA.

In Figure 4, we present an example of the resulting values of $\mathrm{O}\left\{\tilde{\chi}_{g}\right\}$ for the case when the search space is split into 6 sectors. Comparing the average and the minimum values of $\mathrm{O}\left\{\breve{\chi}_{g}\right\}$ among the sectors with the ones achieved by the Gaussian matrix (see Table I) we can see that the proposed technique outperforms its random counterpart.

\section{CONCLUSION}

In this work we studied the design of the measurement matrix for compressive 3-D DoA estimation with sensor arrays. We propose to choose the measurement matrix such that it minimizes the Cramr-Rao Lower Bound (CRLB) for estimating the DoAs from the compressed observations. Moreover, we demonstrate that this target function admits a closed-form solution which provides the CRLB-optimal measurements with 


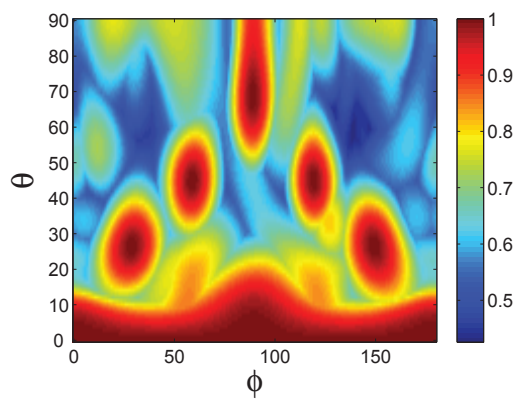

Fig. 4. $\mathrm{O}\left\{\breve{\chi}_{g}\right\}$ as a function of $\theta$ and $\phi$ when $N_{S}=6$.

TABLE I

THE VALUE OF $O\left\{\breve{\chi}_{g}\right\}$.

\begin{tabular}{c|c|c} 
& Average & Minimum \\
\hline $\begin{array}{c}\text { The proposed } \\
\text { surveillance technique }\end{array}$ & 0.72 & 0.42 \\
\hline $\begin{array}{c}\text { Surveillance with } \\
\text { random Gaussian matrix }\end{array}$ & 0.27 & 0.16
\end{tabular}

only $M=2$ compressed channels. We apply this strategy to two distinct applications such as detection of new targets and tracking of the previously identified ones. Presented numerical results demonstrate that application of the proposed approach allows to significantly improve DoA estimation performance compared to that provided by the commonly considered random matrix designs.

\section{APPENDIX}

\section{DERIVATION OF THE CRLB FOR A SINGLE SOURCE}

We begin by vectorizing the set of measurements $\boldsymbol{Y}$ :

$$
\boldsymbol{y}=\operatorname{vec}(\boldsymbol{Y})=\left(\mathbf{I}_{L} \otimes \boldsymbol{W}\right) \cdot(\boldsymbol{\Psi}(\boldsymbol{\chi}, \boldsymbol{P}, \boldsymbol{t}) \cdot \boldsymbol{s}+\boldsymbol{n}),
$$

where $\boldsymbol{\Psi}(\boldsymbol{\chi}, \boldsymbol{P}, \boldsymbol{t})=e^{j \omega \boldsymbol{t}} \otimes e^{j \omega \boldsymbol{\tau}(\boldsymbol{\chi})}, \boldsymbol{t}=\left[t_{1}, t_{2}, \cdots, t_{L}\right]^{\mathrm{T}}$ and $\otimes$ denotes the Kronecker product.

Assuming that $\boldsymbol{W} \boldsymbol{W}^{\mathrm{H}}=\mathbf{I}_{M}$, one can show that $\boldsymbol{y} \sim$ $\mathcal{N}\left(\boldsymbol{\mu}_{y}, \boldsymbol{\Sigma}_{y}\right)$, where $\boldsymbol{\mu}_{y}=\boldsymbol{W} \cdot e^{j \omega \boldsymbol{\tau}(\boldsymbol{\chi})}$ and $\boldsymbol{\Sigma}_{y}=\sigma_{n}^{2} \cdot \mathbf{I}_{M L}$. Then, the Fisher Information Matrix $\boldsymbol{J}$ can be written as [13]:

$$
\boldsymbol{J}=c_{0}\left[\begin{array}{cc}
e_{\theta}(\boldsymbol{W}, \boldsymbol{\chi}) & e_{\theta \phi}(\boldsymbol{W}, \boldsymbol{\chi}) \\
e_{\theta \phi}(\boldsymbol{W}, \boldsymbol{\chi}) & e_{\phi}(\boldsymbol{W}, \boldsymbol{\chi})
\end{array}\right]
$$

where

$$
\begin{aligned}
& c_{0} \cdot \boldsymbol{e}_{\theta}(\boldsymbol{W}, \boldsymbol{\chi})=\frac{\partial \boldsymbol{\mu}_{y}^{\mathrm{H}}}{\partial \theta} \frac{\partial \boldsymbol{\mu}_{y}}{\partial \theta}= \\
& =\frac{|s|^{2}}{\sigma_{n}^{2}}\left(\left(e^{j \omega t}\right)^{\mathrm{H}} \otimes\left(\boldsymbol{W} \frac{\partial e^{j \omega \boldsymbol{\tau}(\boldsymbol{\chi})}}{\partial \theta}\right)^{\mathrm{H}}\right)\left(e^{j \omega \boldsymbol{t}} \otimes\left(\boldsymbol{W} \frac{\partial e^{j \omega \boldsymbol{\tau}(\boldsymbol{\chi})}}{\partial \theta}\right)\right) \\
& =\frac{L|s|^{2}}{\sigma_{n}^{2}}\left(\frac{\partial e^{j \omega \boldsymbol{\tau}(\boldsymbol{\chi})}}{\partial \theta}\right)^{\mathrm{H}} \boldsymbol{W}^{\mathrm{H}} \boldsymbol{W} \frac{\partial e^{j \omega \boldsymbol{\tau}(\boldsymbol{\chi})}}{\partial \theta}=\boldsymbol{a}(\boldsymbol{\chi})^{\mathrm{H}} \boldsymbol{W}^{\mathrm{H}} \boldsymbol{W} \boldsymbol{a}(\boldsymbol{\chi}),
\end{aligned}
$$

and $\boldsymbol{a}(\boldsymbol{\chi})=\frac{\partial e^{j \omega \boldsymbol{\tau}(\boldsymbol{\chi})}}{\partial \theta}$. Similarly, it can be shown that

$$
\begin{gathered}
\boldsymbol{e}_{\phi}(\boldsymbol{W}, \boldsymbol{\chi})=\frac{\partial \boldsymbol{\mu}_{y}^{\mathrm{H}}}{\partial \phi} \frac{\partial \boldsymbol{\mu}_{y}}{\partial \phi}=\boldsymbol{b}(\boldsymbol{\chi})^{\mathrm{H}} \boldsymbol{W}^{\mathrm{H}} \boldsymbol{W} \boldsymbol{b}(\boldsymbol{\chi}), \\
\boldsymbol{e}_{\theta \phi}(\boldsymbol{W}, \boldsymbol{\chi})=\frac{\partial \boldsymbol{\mu}_{y}^{\mathrm{H}}}{\partial \theta} \frac{\partial \boldsymbol{\mu}_{y}}{\partial \phi}=\boldsymbol{a}(\boldsymbol{\chi})^{\mathrm{H}} \boldsymbol{W}^{\mathrm{H}} \boldsymbol{W} \boldsymbol{b}(\boldsymbol{\chi}),
\end{gathered}
$$

where $\boldsymbol{b}(\boldsymbol{\chi})=\frac{\partial e^{j \omega \boldsymbol{\tau}(\boldsymbol{\chi})}}{\partial \phi}$.

Suppose that $\boldsymbol{\zeta}(\boldsymbol{\chi})=\left[\begin{array}{c}-\sin \left(\theta_{k}\right) \cos \left(\phi_{k}\right) \\ -\sin \left(\theta_{k}\right) \sin \left(\phi_{k}\right) \\ -\cos \left(\theta_{k}\right)\end{array}\right]$. Then, for a symmetric array we can write the following

$$
\begin{aligned}
\boldsymbol{a}(\boldsymbol{\chi})^{H} \boldsymbol{b}(\boldsymbol{\chi}) & ==\left(\frac{\partial e^{j \omega \boldsymbol{\tau}(\boldsymbol{\chi})}}{\partial \theta}\right)^{H} \frac{\partial e^{j \omega \boldsymbol{\tau}(\boldsymbol{\chi})}}{\partial \theta} \\
& =\left(e^{j \omega \boldsymbol{\tau}(\boldsymbol{\chi})}\right)^{H} \operatorname{diag}\left(\boldsymbol{P} \frac{\partial \boldsymbol{\zeta}(\boldsymbol{\chi})}{\partial \theta}\right) \operatorname{diag}\left(\boldsymbol{P} \frac{\partial \boldsymbol{\zeta}(\boldsymbol{\chi})}{\partial \phi}\right) e^{j \omega \boldsymbol{\tau}(\boldsymbol{\chi})} \\
& =\left(e^{j \omega \boldsymbol{\tau}(\boldsymbol{\chi})}\right)^{H} \boldsymbol{D} e^{j \omega \boldsymbol{\tau}(\boldsymbol{\chi})}=\operatorname{Tr}\{\boldsymbol{D}\}=0
\end{aligned}
$$

\section{REFERENCES}

[1] A. Gurbuz, J. McClellan, and V. Cevher, "A compressive beamforming method," in IEEE International Conference on Acoustics, Speech and Signal Processing, March 2008, pp. 2617-2620.

[2] O. Teke, A. C. Gurbuz, and O. Arikan, "A robust compressive sensing based technique for reconstruction of sparse radar scenes," Digital Signal Processing, vol. 27, pp. 23-32, 2014.

[3] F. Roemer, M. Ibrahim, R. Alieiev, M. Landmann, R. S. Thomae, and G. D. Galdo, "Polarimetric compressive sensing based doa estimation," in 18th International ITG Workshop on Smart Antennas (WSA). VDE, 2014, pp. 1-8.

[4] J.-F. Gu, W.-P. Zhu, and M. Swamy, "Compressed sensing for doa estimation with fewer receivers than sensors," in Circuits and Systems (ISCAS), 2011 IEEE International Symposium on. IEEE, 2011, pp. $1752-1755$.

[5] J. M. Duarte-Carvajalino, G. Yu, L. Carin, and G. Sapiro, "Task-driven adaptive statistical compressive sensing of Gaussian mixture models," IEEE Transactions on Signal Processing, vol. 61, no. 3, pp. 585-600, 2013.

[6] M. Ibrahim, F. Roemer, and G. Del Galdo, "On the design of the measurement matrix for compressed sensing based doa estimation," in Acoustics, Speech and Signal Processing (ICASSP), 2015 IEEE International Conference on. IEEE, 2015, pp. 3631-3635.

[7] Y. Yu, A. P. Petropulu, and H. V. Poor, "Measurement matrix design for compressive sensing-based MIMO radar," IEEE Transactions on Signal Processing, vol. 59, no. 11, pp. 5338-5352, 2011.

[8] J. Zhang, D. Zhu, and G. Zhang, "Adaptive compressed sensing radar oriented toward cognitive detection in dynamic sparse target scene," IEEE Transactions on Signal Processing, vol. 60, no. 4, pp. 1718-1729, 2012.

[9] J. Mairal, F. Bach, and J. Ponce, "Task-driven dictionary learning," IEEE Transactions on Pattern Analysis and Machine Intelligence, vol. 34, no. 4, pp. 791-804, April 2012.

[10] A. Poudel and D. R. Fuhrmann, "Adaptive sensing and target tracking of a simple point target with online measurement selection," in Conference Record of the Forty Fourth Asilomar Conference on Signals, Systems and Computers (ASILOMAR), 2010, pp. 2017-2020.

[11] M. Sharp, M. Pekala, J. Nanzer, I.-J. Wang, D. Lucarelli, and K. Lauritzen, "Exploiting adaptive beamforming for compressive measurements," in IEEE 7th Sensor Array and Multichannel Signal Processing Workshop (SAM), 2012, pp. 337-340.

[12] T. Kanungo, D. M. Mount, N. S. Netanyahu, C. D. Piatko, R. Silverman, and $\mathrm{A}$. Y. Wu, "An efficient k-means clustering algorithm: analysis and implementation," IEEE Transactions on Pattern Analysis and Machine Intelligence, vol. 24, no. 7, pp. 881-892, 2002.

[13] M. K. Steven, "Fundamentals of statistical signal processing," PTR Prentice-Hall, Englewood Cliffs, NJ, 1993. 